\title{
Activated transport through a quantum dot with extended edge channels
}

\author{
I. K. Marmorkos and C. W. J. Beenakker \\ Instituut-Lorentz, University of Leiden, P.O. Box 9506, 2300 RA Leiden, The Netherlands
}

(Received 18 August 1992)

\begin{abstract}
We study the Coulomb-blockade oscillations in the conductance of a quantum dot in the quantum Hall effect regime. Our model calculation generalizes the self-consistent Thomas-Fermi approach of McEuen et al. for isolated dots, to include extended as well as localized edge states. We find that a Coulomb blockade can exist for the transfer of an electron from an extended to a localized edge state, in accordance with recent experiments by Alphenaar et al. We demonstrate the crucial role played by the incompressibility of the extended edge states, and predict that the conductance oscillations will be suppressed at lower temperatures when an odd rather than an even number of extended edge channels is present.
\end{abstract}

Conduction through a small confined region in a twodimensional electron gas (a "quantum dot") is activated, due to the Coulomb interactions which impose an energy barrier for changing the number of electrons in the dot. This is the Coulomb blockade of single-electron tunneling. ${ }^{1}$ At particular values of the Fermi energy $E_{F}$ of the adjacent electron reservoirs the activation energy vanishes, and resonant tunneling through the quantum dot results in a peak in the conductance. The periodic modulation of the activation energy as a function of $E_{F}$ is observed as a periodic oscillation of the conductance (Coulomb-blockade oscillations). ${ }^{2}$ At the conductance minima, the activation energy $E_{\text {act }}$ takes on its largest value, given by the charging energy $E_{c}=e^{2} / 2 C$, with $C$ the classical capacitance between the confined region and the reservoirs. A key assumption of this "orthodox model" 1 of the Coulomb blockade is that the conductance $G_{B}$ of the barrier between the dot and the reservoirs is smaller than the conductance quantum $e^{2} / h$, so that the number of electrons in the quantum dot is a sharply defined classical variable that can take on only integer values. For $G_{B}>e^{2} / h$ no Coulomb blockade is expected classically.

The situation is different in a strong magnetic field, in the regime of the quantum Hall effect. In that regime conductance occurs via edge states circulating along the circumference of the quantum dot. Edge states with the same Landau-level quantum number form an edge channel. If $G_{B}<e^{2} / h$ all edge states are localized in the quantum dot, which is completely isolated from the electron reservoirs [see Fig. 1(a)]. In that case the main modification of the orthodox model is that the charging energy is no longer well described by the classical capacitance, because of the large screening length in the quantum Hall effect regime. McEuen et $a l^{3}{ }^{3}$ have introduced an improved model which takes into account charging effects self-consistently, within the Thomas-Fermi approximation. This model was used successfully to explain experiments on Coulomb-blockade oscillations in isolated quantum dots. ${ }^{3}$ If $G_{B}>e^{2} / h$ some edge states in the quantum dot extend into the reservoirs [Fig. 1(b)], so that the number of electrons in the dot is not restricted to have in- teger values and one would expect no Coulomb blockade to occur. Recently, however, Alphenaar et al. ${ }^{4}$ observed Coulomb regulated conductance oscillations even in the presence of extended edge channels $\left(G_{B}>e^{2} / h\right)$. They attributed these conductance oscillations to a Coulomb blockade for tunneling from an extended to a localized edge channel. For some values of $E_{F}$ the Coulomb blockade is removed, so that electrons can tunnel resonantly between the extended edge states at the upper and lower edge via an intermediate localized edge state. This is a mechanism for resonant backscattering, ${ }^{5}$ which leads to a conductance minimum. In this way the conductance oscillations would originate from an oscillatory activation energy for adding a single electron to a localized edge channel.

In this paper, motivated by the experimental work of Alphenaar et al., ${ }^{4}$ we study theoretically the problem of the Coulomb blockade in the regime of extended edge channels. By extending the self-consistent model proposed by McEuen et al., ${ }^{3}$ we calculate the activation en-

(a)

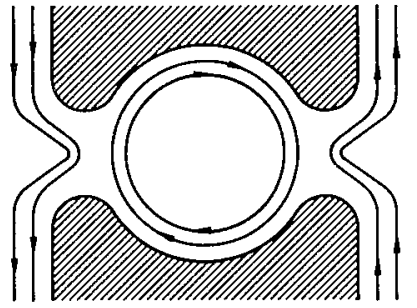

(b)

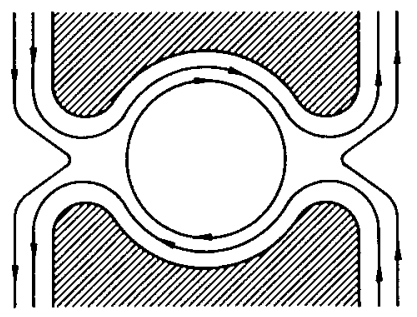

FIG. 1. Schematic view of a quantum dot with (a) no extended edge channels and (b) with one extended and one localized edge channel. 
ergy for tunneling from one extended to one localized edge channel. We find an oscillatory activation energy as a function of $E_{F}$, the periodicity of which equals the periodicity of the conductance oscillations as a function of Fermi energy, whereas the amplitude can be measured from the temperature dependence of the oscillations. ${ }^{6}$ For comparison we also calculate the activation energy for the case that both edge channels are localized (i.e., for the usual case of an isolated quantum dot). An interesting picture of an interacting dot with extended edge states is revealed with a crucial role played by the incompressibility of the extended states, which makes the charging energy insufficient to determine alone the activation energy of the quantum dot.

Our model generalizes that of McEuen et al., ${ }^{3}$ to include extended as well as localized edge channels. The starting point is the energy functional

$$
\begin{aligned}
U= & \sum_{n, s}\left[\omega_{c}\left(n+\frac{1}{2}\right)+g \mu_{B} B s\right] N_{n s}+\int d^{2} \mathbf{r} V_{\text {ext }}(\mathbf{r}) \rho(\mathbf{r}) \\
& +\frac{1}{2} \int d^{2} \mathbf{r} \int d^{2} \mathbf{r}^{\prime} V_{e e}\left(\mathbf{r}-\mathbf{r}^{\prime}\right) \rho(\mathbf{r}) \rho\left(\mathbf{r}^{\prime}\right)
\end{aligned}
$$

The index $n=0,1,2, \ldots$ labels the Landau levels, the index $s= \pm \frac{1}{2}$ labels the spin polarization. The sum over $n$ and $s$ gives the kinetic and Zeeman energy of the $N_{n s}$ electrons in each Landau level $\left[\omega_{c}=e B / m\right.$ is the cyclotron frequency and $g \mu_{B} B$ the Zeeman splitting, in a magnetic field $B$ perpendicular to the two-dimensional electron gas (2DEG)]. The integrals over the areal electron density $\rho(\mathbf{r})$ give the confinement and interaction energy in the approximation of a slowly varying electron density. We take a parabolic confining potential $V_{\text {ext }}(r)=\frac{1}{2} m \omega_{0}^{2} r^{2}$. Near the tunnel barriers, where the extended edge channels join with the electron reservoirs, the external potential should have a saddle point, which we have not included. Although the saddle point is crucial for calculating the transmission probabilities, it will have little effect on the ground-state energy if the lateral extension of the tunnel barriers is much smaller than that of the quantum dot. As in Ref. 3, the electron-electron interaction potential is modeled by

$$
V_{e e}(r)=\frac{e^{2}}{\epsilon}\left[\left(r^{2}+\delta^{2}\right)^{-1 / 2}-\left(r^{2}+4 d^{2}\right)^{-1 / 2}\right]
$$

to include the effects of the finite thickness $\delta$ of the 2DEG layer and the image charge on a gate electrode at a distance $d$ above the 2DEG. In our numerical work we took $\hbar \omega_{0}=0.8 \mathrm{meV}, \delta=50 \AA, d=100 \AA$, and dielectric constant $\epsilon=13.6$ appropriate for GaAs-based devices. Our general conclusions are not sensitive to the specific form of the interaction and confining potentials.

To determine the ground state of the quantum dot in equilibrium with electron reservoirs at Fermi energy $E_{F}$, we minimize the thermodynamic potential $\Omega=U-N E_{F}$, where $N=\int d^{2} \mathbf{r} \rho(\mathbf{r})$ is the number of electrons in the dot. The number of electrons with quantum numbers $n$, $s$ is given by $N_{n s}=\int d^{2} \mathbf{r} \rho_{n s}(\mathbf{r})$, with $N=\sum_{n s} N_{n s}$. The number $N_{n s}$ is constrained to be an integer for a localized edge channel, whereas it is an unconstrained positive real number for an extended edge channel. The
Landau-level degeneracy constrains the particle density $\rho_{n s}(\mathbf{r})$ of electrons with quantum numbers $n, s$ to the interval $0 \leq \rho_{n, s}(\mathbf{r}) \leq \frac{e B}{h}$. The minimization of $\Omega$ subject to the above constraints is carried out numerically, and yields a ground-state thermodynamic potential $\Omega_{g}$, with the corresponding density distributions per Landau level. To obtain the activation energy $E_{\text {act }}$ we repeat the minimization twice, subject to the additional constraint that the total number of electrons in the localized edge channels is either one more or one less than the number $N_{0}$ in the ground-state configuration. This yields two additional thermodynamic potentials, $\Omega_{+}$and $\Omega_{-}$. The activation energy is defined by $E_{\text {act }}=\min \left(\Omega_{+}-\Omega_{g}, \Omega_{-}-\Omega_{g}\right)$, and is non-negative by construction. If $E_{\text {act }}=0$, either the process $N_{0} \rightarrow N_{0}+1 \rightarrow N_{0} \rightarrow \cdots$ or the process $N_{0} \rightarrow N_{0}-1 \rightarrow N_{0} \rightarrow \cdots$ costs zero energy. According to the resonant backscattering mechanism discussed above, this corresponds to a conductance minimum. If $E_{\text {act }}>0$, backscattering is suppressed at low temperatures (when $k_{B} T \ll E_{\text {act }}$ ).

In Fig. 2 we plot $E_{\text {act }}$ as a function of $E_{F}$ for a quantum dot with one extended and one localized edge channel at $B=2 \mathrm{~T}$. Both edge channels have the same Landau in$\operatorname{dex}(n=0)$, but opposite spin polarization $\left(s= \pm \frac{1}{2}\right)$. We define the incompressibility energy $E_{\text {incomp }}$ as the minimum energy required to excite an electron from an extended to a localized edge channel without changing the electron density distribution $\rho(\mathbf{r})$. In the case of Fig. 2 , or, more generally, if the number $n_{\text {ext }}$ of extended edge channels is odd, we have $E_{\text {incomp }}$ equal to the Zeeman splitting $g \mu_{B} B$. If $n_{\text {ext }}$ is even, on the other hand, the incompressibility energy is determined by the cyclotron energy $\hbar \omega_{c}$, or more precisely $E_{\text {incomp }}=\hbar \omega_{c}-g \mu_{B} B$. By definition, $E_{\text {incomp }} \geq E_{\text {act }}$. As we now demonstrate,

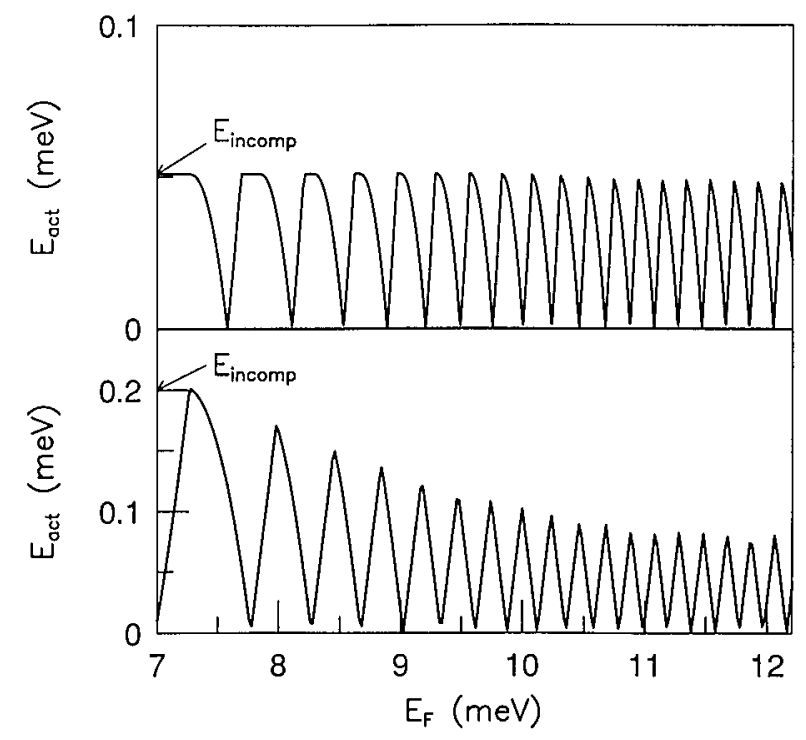

FIG. 2. Activation energy as a function of Fermi energy for a quantum dot with two edge channels occupied, one extended and one localized. The incompressibility energy $E_{\text {incomp }}$ equals the Zeeman splitting $g \mu_{B} B$ at $B=2 \mathrm{~T}$. In the top panel $g=0.44$. In the bottom panel $g$ is multiplied by a factor of 4 to demonstrate the effect of $E_{\text {incomp }}$ on $E_{\text {act }}$. 
the incompressibility energy plays a crucial role in this problem (which it did not in the case of an isolated system considered previously). The two panels in Fig. 2 both show a periodic modulation of $E_{\text {act }}$, with the same periodicity but different amplitude. In the top panel the $g$ factor was set at the value $g_{0}=0.44$ appropriate for $\mathrm{GaAs}$, while in the bottom panel we set $g=4 g_{0}$ to artificially increase the incompressibility energy. The value $g_{0}=0.44$ is the bare $g$ factor. Whether or not exchange effects enhance the $g$ factor in a confined geometry is a matter of some debate, which we do not consider here. In the top panel the activation energy is shortcircuited exactly at the level of the incompressibility energy. The energy scale for activated transport is thus set
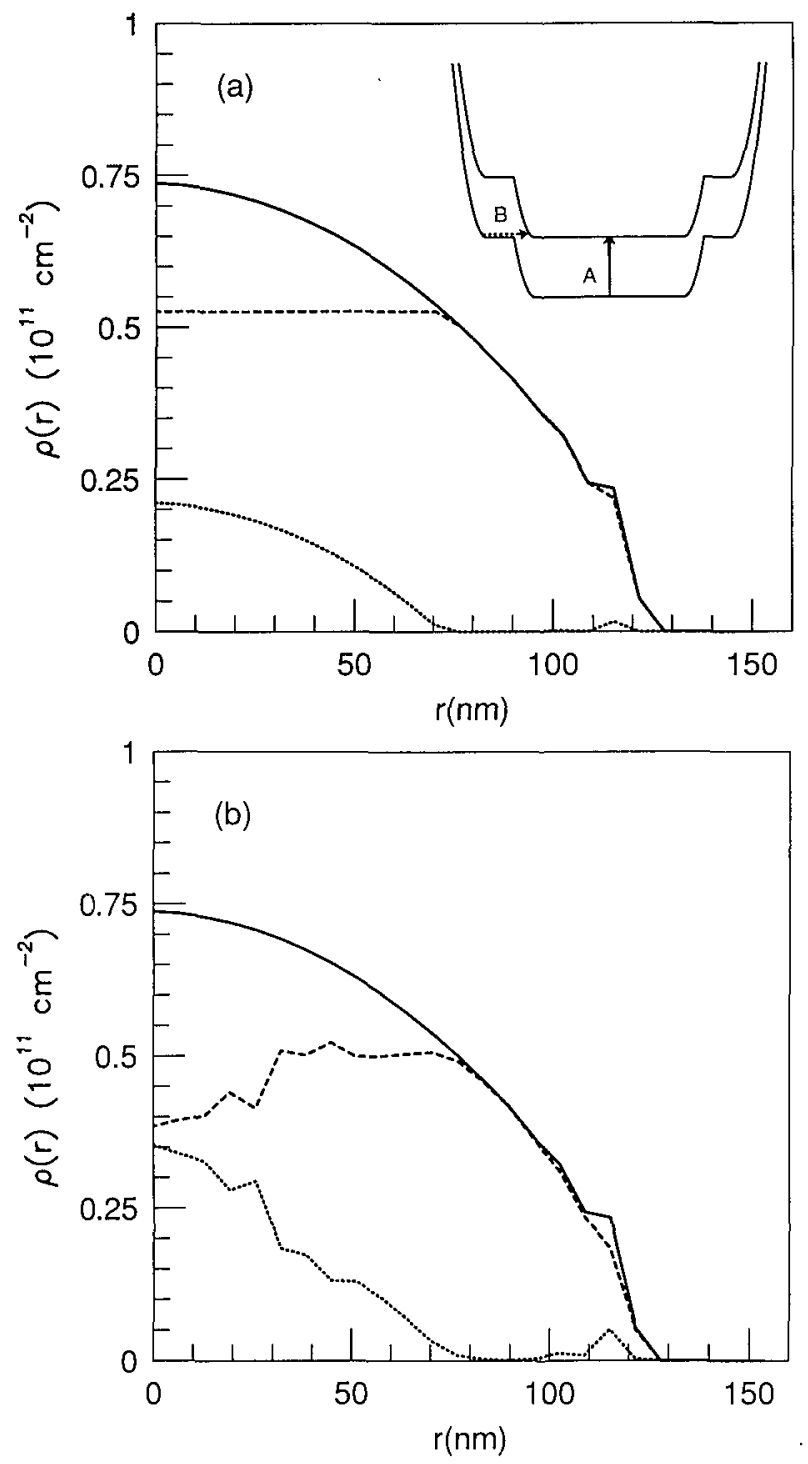

FIG. 3. Density distribution $\rho(r)$ at $E_{F}=8.24 \mathrm{meV}$, corresponding to a plateau region in the top panel of Fig. 2. The dashed curve is the density profile of the lowest (extended) edge channel, the dotted one of the highest (localized) edge channel, and the solid curve is the total electron density. The curves in (a) refer to the ground state and those in (b) to the excited state, which involves the transfer of an electron from the lower to the higher Landau level. by the Zeeman energy and not by the charging energy $E_{c}$, hence there is no Coulomb blockade. The existence of a Coulomb blockade for transport from an extended to a localized edge channel requires $E_{\text {incomp }}>E_{c}$, as in the bottom panel of Fig. 2. In general, this will be the case if $E_{\text {incomp }}$ is determined by the cyclotron energy, i.e., if $n_{\text {ext }}$ is even. To summarize, we predict a smaller activation energy (and hence a stronger temperature dependence of the conductance oscillations) if an odd, rather than an even, number of extended edge channels is present in the quantum dot.

This is our main conclusion. We now discuss Fig. 2 in more detail. Consider first the top panel. The oscillations in $E_{\text {act }}$ have a triangular shape, which is truncated at $E_{\text {incomp }}=0.051 \mathrm{meV}$. At these maximum values of the activation energy, equal to the Zeeman splitting, the activated process consists of the transfer of one electron from the lower (extended) to the higher (localized) edge channel. The charge transfer takes place in the same region in space, without charge separation, so that the change in electrostatic energy is zero. This can be seen more clearly in Fig. 3, where we plot the density profile in the dot before and after the electron transfer to the higher Landau level. The electron transfer breaks down the incompressibility of the lowest Landau level, but does not change the net density profile. The plateaus at the maxima of $E_{\text {act }}$ occur because, over a range of Fermi energies, it is more advantageous energetically to excite an electron from the lowest to the higher Landau level in the same region in space [process $A$ in the inset of Fig. 3(a)], rather than to transport the electron from one Landau level to the other across an incompressible region (process $B$ ). The second process is more advantageous in the bottom panel of Fig. 2, where $E_{\text {act }}$ is not truncated by $E_{\text {incomp }}=0.20 \mathrm{meV}$, but reaches a maximum determined by the electrostatic (charging) energy.

For comparison we have also calculated the activation

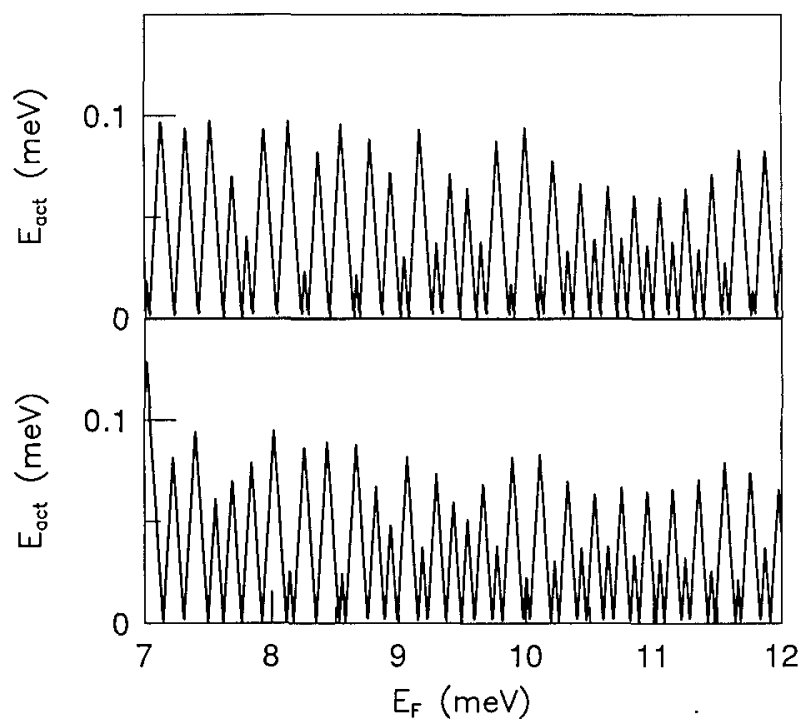

FIG. 4. Activation energy as a function of Fermi energy for an isolated quantum dot with two localized edge channels. Same parameter values as in Fig. 2. 
energy when both edge channels are localized. This is the case of an isolated dot studied by McEuen et al. ${ }^{3}$ (who did not, however, present results for the activation energy). In this case $E_{\text {act }}$ is the activation energy for tunneling from one reservoir to the other via the quantum dot. A minimum of $E_{\text {act }}$ now corresponds to a maximum in the conductance. Results are shown in Fig. 4. As in Fig. 2, the top panel is for $g=g_{0}$ and the bottom panel for $g=4 g_{0}$. In contrast to Fig. 2, the change in $E_{\text {incomp }}$ has no effect on the average peak height, but it does influence the detailed structure of the oscillations.

To obtain a qualitative understanding of the rich structure in Fig. 4, we proceed as follows. We first note that, since $d E_{\text {act }} / d E_{F}= \pm 1$, there is a simple linear relationship between the heights of the peaks and their spacing. It is therefore sufficient to consider the spacing of the peaks, or equivalently of the points of zero activation energy. To this end we examine the dependence on the reservoir Fermi energy $E_{F}$ of the ground-state occupation numbers $N_{1}\left(E_{F}\right)$ and $N_{2}\left(E_{F}\right)$ of the two edge channels. Zero activation energy occurs when either $N_{1}$ or $N_{2}$ changes by one. Numerically, we found that over a large range of Fermi energies an increment in $N_{1}$ alternates with one in $N_{2}$. This "cyclic depopulation" of the Landau levels is in agreement with recent experimental observations. ${ }^{7}$ We can thus represent the two functions $N_{1}\left(E_{F}\right)$ and $N_{2}\left(E_{F}\right)$ by two staircases of unit step height and approximately equal step width $\Delta E_{F}$, but phase shifted by some arbitrary amount $\delta E_{F}$ between 0 and $\Delta E_{F}$. This leads directly to a doublet structure of the peaks in the activation energy, with alternating small and large spacings (and peak heights) in a ratio of $\delta E_{F}:\left(\Delta E_{F}-\delta E_{F}\right)$. Such a doublet structure is a striking feature of our numerical calculations in Fig. 4. The slow modulation ("beating") superimposed on the doublet structure can be interpreted as arising from a small difference in step width for $N_{1}\left(E_{F}\right)$ and $N_{2}\left(E_{F}\right)$. This also implies that occasionally the cyclic depopulation skips a Landau level, which is indeed what we find in the calculation. Our conclusion here is that the strong variation in peak height in Fig. 4 occurs not because the capacitive energies of the two edge channels are very different, but because they are very similar.

We thank B. W. Alphenaar for communicating his experimental data to us prior to publication. This work was supported by the Dutch Science Foundation NWO/FOM.
${ }^{1}$ D. V. Averin and K. K. Likharev, in Mesoscopic Phenomena in Solids, edited by B. L. Al'tshuler, P. A. Lee, and R. A. Webb (North-Holland, Amsterdam, 1991).

${ }^{2}$ For a review see $\mathrm{H}$. van Houten, C. W. J. Beenakker, and A. A. M. Staring, in Single Charge Tunneling, Vol. 294 of NATO Advanced Study Institute, Series B: Physics, edited by H. Grabert and M. H. Devoret (Plenum, New York, 1992). ${ }^{3}$ P. L. McEuen, E. B. Foxman, J. Kinaret, U. Meirav, M. A. Kastner, N. S. Wingreen, and S. J. Wind, Phys. Rev. B 45, 11419 (1992).
${ }^{4}$ B. W. Alphenaar, A. A. M. Staring, H. van Houten, M. A. A. Mabesoone, O. J. A. Buyk, and C. T. Foxon, Phys. Rev. B 46, 7236 (1992).

${ }^{5}$ J. K. Jain, Phys. Rev. Lett. 60, 2074 (1988).

${ }^{6}$ S. B. Field, M. A. Kastner, U. Meirav, J. H. F. ScottThomas, D. A. Antoniadis, H. I. Smith, and S. J. Wind, Phys. Rev. B 42, 3523 (1990).

${ }^{7}$ A. A. M. Staring, B. W. Alphenaar, H. van Houten, L. W. Molenkamp, O. J. A. Buyk, M. A. A. Mabesoone, and C. T. Foxon, Phys. Rev. B 46, 12869 (1992). 